\title{
Televisión Comunitaria: Factores de Éxito para el Crecimiento en Ecuador
}

\section{Community Television: Success Factors for Growth in Ecuador}

\author{
Noboa Auz, Marjorie Ligena ${ }^{1 *}$, Loor Saltos, Mónica Guisella ${ }^{1}$, Del Pozo, Víctor H. ${ }^{1}$ \\ ${ }^{1}$ Universidad de Guayaquil \\ *marjorie.noboaa@ug.edu.ec
}

DOI: https://doi.org/10.26871/killkana_social.v2i1.181

\begin{abstract}
Resumen
Se han creado tres televisoras comunitarias en Ecuador desde la promulgación de la ley de comunicación en el 2013 hasta la fecha. Dos de ellas, dedicadas a la identidad cultural religiosa, y una, a generar identidad cultural indígena. El objetivo del presente estudio es identificar factores de éxito adoptados en otros países que puedan aportar al desarrollo y crecimiento de la televisión comunitaria en Ecuador. Para la presente investigación se seleccionaron artículos científicos de revistas de alto impacto con estudios de televisión comunitaria en América Latina, Asia y Europa realizados en los últimos cinco años. Se realizó una revisión descriptiva de los aspectos más relevantes resultantes de los estudios y se compararon con investigaciones realizadas en Ecuador. La percepción de la audiencia es que la televisión comunitaria les genera identidad cultural, pero no se sienten identificados con los presentadores ni periodistas del canal. Como conclusión se identificaron y describieron algunos aspectos claves que pueden beneficiar a la televisión comunitaria ecuatoriana como: (a) las alianzas con otras televisoras comunitarias, proveedoras de cable, interface y televisoras locales, (b) ampliación a medios digitales, (c) basar los contenidos comunitarios en las funciones lingüísticas emotivas y apelativas para fidelizar a la audiencia, y (d) los contenidos deben reflejar la vida diaria de la audiencia.
\end{abstract}

Palabras clave: contenidos, identidad cultural, televisión comunitaria .

\begin{abstract}
Three community television stations have been created in Ecuador since the enactment of the communication law in 2013 to date. Two of them dedicated to religious cultural identity and one to generate indigenous cultural identity. The objective of this study is to identify success factors adopted in other countries that can contribute to the development and growth of community television in Ecuador. For the present investigation, scientific articles from high impact journals were chose with community television studies in Latin America, Asia and Europe conducted in the last five years. A descriptive review of the most relevant aspects resulting from the studies was carried out and compared with investigations carried out in Ecuador. The perception of the audience is that community television generates cultural identity, but they do not feel identified with the presenters or journalists of the channel. In sum, some key aspects that could benefit Ecuadorian community television were identified and described as: (a) alliances with other community television, cable, interface and local television providers, (b) expansion to digital media, (c) base community content in emotive and appealing linguistic functions to make the audience more loyal, and $(d)$ the contents must reflect the daily life of the audience.
\end{abstract}

Key words: community television, content, cultural identity.

\section{Introducción}

En América Latina en los años 1980 aparecieron las primeras experiencias de comunicación comunitaria, promoviendo la identidad cultural y el desarrollo socioeconómico de cada comunidad. La revisión de la literatura demuestra que existen factores que mejorar como: la competencia entre medios comunitarios, privados y públicos, los rendimientos sostenibles en el tiempo, el profesionalismo técnico apropiado, la tecnología y la adopción de manejo de medios digitales. En el presente estudio se realizó una investigación sobre las experiencias de las televisoras comunitarias en los últimos cinco años. Se seleccionaron artículos científicos de revistas de alto impacto Web of Science. En el año 2013, Ecuador promulgó leyes que fomentan la creación de medios comunitarios, para mantener la pluriculturalidad y la cultura ancestral. Para la presente investigación se consideró la televisión comunitaria, con el fin de identificar los factores de éxito y ¿qué experiencias de otros países pueden aportar al desarrollo de este medio comunitario en Ecuador? 


\section{Metodología}

Para el desarrollo del presente estudio se seleccionaron las revistas de alto impacto con el buscador Web of Science para asegurar la fiabilidad y validez de los datos, con las palabras claves community television, televisión comunitaria, identidad cultural y televisión comunitaria, cultural identity and community television. Para una mejor calidad de información se utilizó el criterio de tiempo entre los años 2012 - 2017. Para conocer el contexto ecuatoriano se buscó en los repositorios de trabajos de titulación de la Universidad de Guayaquil en la carrera de Comunicación Social en el mismo período de tiempo.

\subsection{Medios Comunitarios}

Los medios comunitarios tienen como objetivo abrir espacios en un determinado grupo o comunidad al ejercicio del derecho de recibir información y a la vez comunicar (Gumucio, 2015). La participación de los habitantes en la creación de programas de televisión permite a los televidentes ser más críticos, fomenta la conciencia y reflexión sobre uno mismo, los demás y la sociedad, y proporciona una base que alienta a individuos y grupos a creer que pueden afectar a la sociedad en general (Higgins, 1999). La definición de comunidad: en estas nuevas formas de conectividad globalizadas, consiste en un grupo de personas que tienen intereses o ambiciones similares que comparten una interacción social que es posible por causa de la tecnología por el cyber-espacio virtual (Kozinets, 2010). En el presente estudio se consideró como comunidad a grupos geográficamente unidos que comparten costumbres, tradiciones, lenguaje, creencias y cultura.

\section{Identidad Cultural}

Una comunidad no sólo es un grupo de personas sino también la intervención de factores como el sentido de pertenencia, el patrimonio, los valores y las creencias que construyen la identidad cultural, la agrupación de algunos elementos permite que los grupos se reconozcan entre sí, estas fuentes de sentido de pertenencia se van construyendo de forma globalizada. (Terry, 2011; Ruiz, Calderòn, y Torres, 2011). La cultura se construye a través de los hábitos y el contexto en el cual esta inmiscuida una comunidad, pudiendo tomar decisiones para lograr mejorar la eficiencia y bienestar de los individuos (Aguilar, 2013). La identidad cultural no es estática pues los procesos históricos como la interacción de la vida cotidiana construyen constantemente intercambios sociales y culturales entre las personas de una misma comunidad, en la búsqueda de identidad interviene lo personal, social y cultural (Marcús, 2011; Mosquera, Rincòn, y Hurtado, 2013).

A pesar de las identidades culturales que cada comunidad mantiene existe un aspecto fundamental que influye en la continuidad de tradiciones ancestrales, como la globalización que permite adquirir nuevas creencias y costumbres diferentes a las tradicionales. La globalización ha provocado nuevas identidades como resultado de la apertura de fronteras, valorando la diversidad pero adaptarse a un mundo global, significa también la pérdida de su identidad cultural y un legado histórico forjado por sus individuos en el pasado (Mora y Villar, 2015). Los medios de comunicación juegan un papel importante en el intercambio de información y flujo de ideas, conocimientos, valores y, por consiguiente, el entorno cultural se transforma aceleradamente (Sandoval, 2009). En el caso del área asiática (Lee, 2004) señaló que los productores de televisión coreana se han inspirado en los dramas televisivos japoneses, sin embargo, no han copiado directamente los programas. Pero (Katsumata, 2012) argumentó que ciertos productos de la cultura popular del Japón está concentrada en el Este de Asia, como la música J-pop y los dramas de televisión, estos productos están influenciando la moda y el estilo de vida de jóvenes en algunos países asiáticos.

Ecuador por ser un país pluricultural y multiétnico se ha institucionalizado que "los idiomas ancestrales son oficiales para los pueblos indígenas en su territorio, el Estado respetará y estimulará su conservación y uso" (Asamblea Nacional Repùblica del Ecuador, 2008, pág. 16). La influencia de otras costumbres y tendencias actuales provenientes de la globalización podrían afectar y hasta olvidar las costumbres y sabidurías ancestrales ecuatorianas que cuentan con una diversidad de pueblos y nacionalidades en sus tres regiones: costa, sierra y oriente, como se detalla en la Tabla 1.

Tabla 1. Pueblos y nacionalidades del Ecuador

\begin{tabular}{|c|c|}
\hline Región & Pueblos y Nacionalidades \\
\hline Costa & $\begin{array}{l}\text { - Awá } \\
\text { - Chachi } \\
\text { - Epera } \\
\text { - Tsá́chila } \\
\text { - Manta } \\
\text { - Huancavilca }\end{array}$ \\
\hline Sierra & $\begin{array}{l}\text { - Karanki } \\
\text { - Ntabuela } \\
\text { - Otavalo } \\
\text { - Kayambi } \\
\text { - Kitu-Kara } \\
\text { - Panzaleo } \\
\text { - Chibuelo } \\
\text { - Salasaka } \\
\text { - Kisapincha } \\
\text { - Kicwa de Tunguragua } \\
\text { - Waranka } \\
\text { - Puruhá } \\
\text { - Kañari } \\
\text { - Saraguro }\end{array}$ \\
\hline & continua en la próxima página \\
\hline
\end{tabular}




\begin{tabular}{|c|c|}
\hline Región & Pueblos y Nacionalidades \\
\hline Amazonia & $\begin{array}{l}\text { - Ái cofán } \\
\text { - Secoya } \\
\text { - Siona } \\
\text { - Huaorani } \\
\text { - Shiwiar } \\
\text { - Zápara } \\
\text { - Achuar } \\
\text { - Shuar } \\
\text { - Kichwa de la amazonia }\end{array}$ \\
\hline $\begin{array}{l}\text { Regiones } \\
\text { Afroecuatorianas }\end{array}$ & $\begin{array}{l}\text { - Costa Norte } \\
\text { - Costa Centro - Sur } \\
\text { - Valle del Chota } \\
\text { - Pichincha } \\
\text { - Sierra Centro - Sur } \\
\text { - Amazonia Norte }\end{array}$ \\
\hline Mestizos & $\begin{array}{l}\text { - Se encuentran distri- } \\
\text { buidas por todo el país }\end{array}$ \\
\hline
\end{tabular}

Fuente: (SIDENPE, 2002) Sistema de indicadores sociales del Ecuador "SIISE"

Con el fin de precautelar las tradiciones ancestrales, (Agencia de Regulación y Control de las Telecomunicaciones, 2013) indica en el artículo 36 que los pueblos y nacionalidades de las distintas etnias de nuestro país (indígenas, afro ecuatorianas y montubias) están en su derecho de difundir y producir contenidos que reflejen su cultura, tradiciones, formas de vida con el fin de fomentar la igualdad y la interculturalidad.

\section{Televisión Comunitaria}

El objetivo de la televisión comunitaria es orientar, educar y entretener a la comunidad, reforzando valores e identidad cultural, difundir contenido local como fiestas, tradiciones, música, con el objetivo de fortalecer y preservar la cultura de una comunidad, esto no significa que nieguen otras culturas, pero si permite tener una afirmación de una identidad cultural propia que muchas veces es negada por los medios masivos (Sánchez, 2012; Márquez, 2012). La comunicación comunitaria tiene que ver con la apropiación del medio, en este sentido la apropiación se entiende como la forma en que la comunidad de manera colectiva desarrolla capacidades para a través del medio se pueda trabajar según sus necesidades (Gumucio, 2011). La televisión comunitaria es el espacio en el que se desenvuelve una determinada comunidad, siendo generadores de ideas siendo participes activos, priorizando su comunidad y transmitiendo costumbres, tradiciones, valores, dando a conocer problemas coyunturales (Márquez, 2013).

\section{Televisión comunitaria en América}

En Colombia el canal comunitario TV Suesca "Nuestra identidad" surgió de la necesidad de sentirse representados y a la vez preservar su identidad cultural, mostrando la cotidiana de la comunidad convirtiéndose en un medio democrático, participativo y cultural (Sànchez y Deaza, 2016). Existen diferentes percepciones en la audiencia radial y televisiva como en el estudio realizado en dos zonas del sur de Santiago de Chile, (Cabalín, 2010) encontró diferencias entre un canal y una radio, los encuestados percibieron que la programación de la radio no responde a sus necesidades, más bien creen que no hay diferencia entre la radio comunitaria y la emisora comercial, por el contrario, del canal comunitario percibieron que en sus contenidos ven expresada su vida diaria y rostros, por lo que configura una representación de esta comunidad que impacta en el proceso de identidad de su audiencia.

La gestión de los factores lugar, espacio e interface son factores esenciales para el éxito de la televisión comunitaria que deben considerar los administradores de estos medios. En el estudio comparativo de políticas y prácticas de TV comunitarias entre Estados Unidos, Reino Unido y Canadá.

Ali (2012) identificó que estas organizaciones se han encontrado con demandas en un mundo digital y las políticas gubernamentales en los medios de televisión comunitaria no corresponden a lo que estos medios experimentan porque no consideran el lugar, espacio y la interface. El lugar se refiere tanto a la infraestructura física como a la participación pública, el espacio es la formación de alianzas con las entidades que promulgan las políticas y los operadores de cable, y la interface en medios digitales, que han permitido a las organizaciones ampliarse más allá de los límites de la televisión por cable para llegar a un público más amplio y participar en nuevas formas de educación (Fuentes-Bautista, 2009).

En 1987 entró la primera televisión comunitaria argentina, en 1984 surgieron en Brasil las primeras experiencias de tv comunitarias. Algunas Tv comunitarias empezaron a reestructurarse para transmitir vía internet a partir del año 2000. Mientras la ley $\mathrm{N}^{\circ} 26.522$ de Argentina reserva el $33 \%$ del espectro radiofónico al sector sin fines de lucro, la ley brasileña otorga el uso de un canal por ciudad de operación de la TV a cable al sector (Sousa, 2016). (Marino, 2012) consideró que el financiamiento es uno de los desafíos centrales de los medios comunitarios, una vez que la ley los reconoce, pero impone una serie de obligaciones a cumplir, como la cuota de contenido propio, infraestructura, pago de derechos de autor y obligaciones laborales.

\section{Televisión comunitaria en Asia y Europa}

Algunos resultados obtenidos de experiencias de la televisión comunitaria fuera de América Latina pueden aportar a identificar factores de éxito imitables. En el estudio de (Shahjahan, Chowdhury, Al-Hadhrami, y Harun, 2016) sobre la educación pre y post natal para disminuir la tasa de mortandad, utilizaron varios medios de transmisión de mensajes para las madres, resultando que el mayor impacto fue enviar mensajes a través de la televisión, ya que el $60.3 \%$ de las madres que vieron la televisión pudieron recibir educación sobre cuidados antenatales y post- 
natales, previniendo complicaciones y asegurando mejores cuidados maternales y salud del niño.

En el estudio comparativo entre cuatro empresas de televisión comunitarias en la India, sólo dos presentaron resultados exitosos,(Chatterjee, 2012) identificó factores a considerar para el éxito: (a) nivel de dificultades para reunir recursos, (b) la elección de programación refleja la afirmación de identidad para pertenecer a un grupo o para separarse de uno, (c) el desarrollo de la televisión comunitaria puede utilizarse para fortalecer asociaciones comunitarias, permite que comunidades más pequeñas tengan acceso a otras comunidades y espacios comunicativos nacionales (d) la expansión de canales locales exitosos incluye a la audiencia nacional no comunitaria.

En Finlandia la falla de la televisión regional y comunitaria se debe a los problemas de organización y financiamiento de actividades y la falta de conocimiento, el desinterés de las personas por el contenido, la falta de rentabilidad y la incertidumbre del mercado (Ojajärvi y Valtonen, 2012). El material ofrecido en la website de la mayoría de las compañías de televisión permiten a los televidentes ver lo que ellos quieren y cuando ellos quieren (Castells, 2009).

\section{La Televisión Comunitaria en Ecuador}

Ecuador promulgó leyes cuyo objetivo es dar espacios para mantener la esencia cultural de cada comunidad. En la (Ley Orgánica de Comunicación, 2013) el artículo 14 acerca del principio de interculturalidad y plurinacionalidad, establece que el Estado promoverá medidas que garanticen la relación intercultural entre comunidades, pueblos y nacionalidades, con el objetivo de producir y difundir contenidos que reflejen la cosmovisión de cada lengua para así de forma progresiva tener en el país una comunicación intercultural. Estableció una distribución equitativa de frecuencias con un $33 \%$ de medios públicos, $33 \%$ medios privados y $34 \%$ medios comunitarios.

En el capítulo 1 artículo 5 (Ley Orgànica de Cultura, 2016) establece que cada comunidad tiene el derecho de mantener y preservar sus tradiciones así como a no ser discriminados por sus creencias y formas de vida. Tanto en la Ley Orgánica de Comunicación como en la Ley de Cultura indica que los medios de comunicación deben promover la identidad cultural.

En la (Agencia de Regulación y Control de las Telecomunicaciones, 2013) artículo 85 se establece que los medios comunitarios corresponden a organizaciones sociales como: comunidades, pueblos o nacionalidades, sin fines de lucro. Los medios comunitarios están dirigidos por y para la comunidad, en el caso de la televisión comunitaria se refiere a productos audiovisuales que cumplan con las necesidades comunicacionales de un grupo social determinado. La difusión y producción de contenidos interculturales hasta el año 2014 en los medios del Ecuador era nula (Andes, 2014).

Ecuador cuenta con tres canales de televisión comunitarios, dos impulsan la cultura religiosa y una de cultura indígena ancestral. La sintonía de la Tv comunitaria indígena llega a un $49 \%$ de la población, sin embargo no se sienten identificados con los presentadores ni periodistas. El 59,89\% sintoniza el canal 47 (tabla 2) de 1 a 2 veces por semana y un $21,47 \%$ sólo el fin de semana. El 36,33\% considera que encuentran información y conocimiento sobre desarrollo económico, social, político y cultural y el $19,53 \%$ encuentra conocimiento sobre el folklore en los contenidos de programación (Loor, 2017).

Los televidentes consideran que los programas generan identidad cultural, a excepción de la programación Centro Naturista, Reencuentro con la salud y Al diálogo con Kleber.

Sobre los atributos que los encuestados perciben acerca de los contenidos, indicaron estar de acuerdo considerando la mediana en el caso de muestran diversidad cultural del país, y promueve la identidad cultural, la mediana se sitúa en 12 y 9 respectivamente, lo que indica que hay una mayor percepción de recibir identidad cultural a través de este canal. En general, lo resultados muestran una aceptación de la audiencia en que los contenidos y programas contienen y generan identidad de su cultura y realidad de su comunidad como se muestran en las Tabla 2 y Tabla 3.

En relación a la percepción de los personajes, conductores y periodistas del canal 47 TV MICC quienes le agradan más y le agrada menos, los participantes contesta-

Tabla 2 Programas del canal 47 TV MICC que fomenta identidad cultural

\begin{tabular}{|c|c|c|c|c|c|c|c|c|c|c|c|}
\hline & $\begin{array}{c}\text { Musica de mi } \\
\text { tierra }\end{array}$ & $\begin{array}{c}\text { Centro } \\
\text { Naturista }\end{array}$ & $\begin{array}{c}\text { Reencuentro } \\
\text { con salud }\end{array}$ & $\begin{array}{l}\text { Al dialogo } \\
\text { con Kleber }\end{array}$ & $\begin{array}{l}\text { Documentale } \\
\text { s TV MICC }\end{array}$ & $\begin{array}{l}\text { Entrevista } \\
\text { "vision 47" }\end{array}$ & Conecta TV & $\begin{array}{c}\text { Noticia } \\
\text { Chaski taki }\end{array}$ & $\begin{array}{c}\text { Costumbres } \\
\text { de mi tierra de } \\
\text { la UTC } \\
\end{array}$ & $\begin{array}{c}\text { Cine } \\
\text { consentido }\end{array}$ & $\begin{array}{c}\text { Voces e } \\
\text { identidad }\end{array}$ \\
\hline Media & 35.40 & 35.40 & 35.40 & 35.40 & 35.40 & 35.40 & 35.40 & 35.40 & 35.40 & 35.40 & 35.40 \\
\hline Error típico & 13.91 & 18.12 & 13.80 & 15.83 & 17.84 & 16.22 & 16.90 & 15.23 & 17.63 & 17.29 & 18.08 \\
\hline Mediana & 26.00 & 22.00 & 28.00 & 19.00 & 16.00 & 16.00 & 13.00 & 15.00 & 19.00 & 13.00 & 12.00 \\
\hline Desviación estándar & 31.11 & 40.53 & 30.85 & 35.39 & 39.88 & 36.26 & 37.78 & 34.05 & 39.42 & 38.67 & 40.43 \\
\hline Coeficiente de asimetría & 0.77 & 1.37 & 0.72 & 0.79 & 1.61 & 0.87 & 0.61 & 0.63 & 1.47 & 0.69 & 0.88 \\
\hline Rango & 72.00 & 98.00 & 76.00 & 80.00 & 99.00 & 81.00 & 76.00 & 75.00 & 96.00 & 83.00 & 89.00 \\
\hline Mínimo & 8.00 & 3.00 & 4.00 & 5.00 & 3.00 & 6.00 & 5.00 & 4.00 & 4.00 & 3.00 & 4.00 \\
\hline Máximo & 80.00 & 101.00 & 80.00 & 85.00 & 102.00 & 87.00 & 81.00 & 79.00 & 100.00 & 86.00 & 93.00 \\
\hline Suma & 177.00 & 177.00 & 177.00 & 177.00 & 177.00 & 177.00 & 177.00 & 177.00 & 177.00 & 177.00 & 177.00 \\
\hline Cuenta & 5.00 & 5.00 & 5.00 & 5.00 & 5.00 & 5.00 & 5.00 & 5.00 & 5.00 & 5.00 & 5.00 \\
\hline Nivel de confianza(95.0\%) & 38.63 & 50.32 & 38.31 & 43.94 & 49.52 & 45.02 & 46.91 & 42.28 & 48.95 & 48.01 & 50.20 \\
\hline
\end{tabular}


Tabla 3 Programas del canal 47 TV MICC que fomenta identidad cultural

\begin{tabular}{|c|c|c|c|c|c|c|c|c|c|c|c|c|}
\hline & $\begin{array}{c}\text { Muestran la } \\
\text { diversidad } \\
\text { cultural del } \\
\text { país }\end{array}$ & $\begin{array}{c}\text { Trans mite } \\
\text { valores éticos }\end{array}$ & $\begin{array}{c}\text { Promueve la } \\
\text { identidad } \\
\text { cultural }\end{array}$ & Informan & Entretienen & Culturizan & Divierten & $\begin{array}{c}\text { Muestran } \\
\text { diversidad } \\
\text { cultural }\end{array}$ & $\begin{array}{c}\text { Ofrece } \\
\text { variedad de } \\
\text { contenidos }\end{array}$ & $\begin{array}{l}\text { Me identifico } \\
\text { culturalmente }\end{array}$ & $\begin{array}{c}\text { Me incentiva } \\
\text { a participar }\end{array}$ & \begin{tabular}{|c} 
Incentiva la \\
participacion \\
de la \\
comunidad \\
\end{tabular} \\
\hline Media & 35.40 & 35.40 & 35.40 & 35.40 & 35.40 & 35.40 & 35.40 & 35.40 & 35.40 & 35.40 & 35.40 & 35.40 \\
\hline Error típico & 19.27 & 12.82 & 18.66 & 17.05 & 12.71 & 21.20 & 10.27 & 20.41 & 11.01 & 17.55 & 17.55 & 12.28 \\
\hline Mediana & 12.00 & 29.00 & 9.00 & 25.00 & 25.00 & 20.00 & 38.00 & 24.00 & 25.00 & 18.00 & 18.00 & 33.00 \\
\hline Desviación estándar & 43.08 & 28.67 & 41.73 & 38.13 & 28.43 & 47.40 & 22.96 & 45.63 & 24.62 & 39.25 & 39.25 & 27.46 \\
\hline Rango & 102.00 & 78.00 & 94.00 & 96.00 & 63.00 & 115.00 & 54.00 & 111.00 & 61.00 & 99.00 & 99.00 & 71.00 \\
\hline Mínimo & 6.00 & 4.00 & 4.00 & 4.00 & 5.00 & 4.00 & 5.00 & 4.00 & 6.00 & 4.00 & 4.00 & 2.00 \\
\hline Máximo & 108.00 & 82.00 & 98.00 & 100.00 & 68.00 & 119.00 & 59.00 & 115.00 & 67.00 & 103.00 & 103.00 & 73.00 \\
\hline Suma & 177.00 & 177.00 & 177.00 & 177.00 & 177.00 & 177.00 & 177.00 & 177.00 & 177.00 & 177.00 & 177.00 & 177.00 \\
\hline Cuenta & 5.00 & 5.00 & 5.00 & 5.00 & 5.00 & 5.00 & 5.00 & 5.00 & 5.00 & 5.00 & 5.00 & 5.00 \\
\hline Nivel de confianza(95.0\%) & 53.49 & 35.59 & 51.81 & 47.34 & 35.30 & 58.86 & 28.51 & 56.66 & 30.57 & 48.74 & 48.74 & 34.10 \\
\hline
\end{tabular}

ron ninguno un $81,36 \%$ y $83,62 \%$ respectivamente (Loor, 2017).

\section{Conclusión}

Considerando que desde la promulgación de la ley de comunicación que asignaba frecuencias para la televisión comunitaria, hasta el año 2018 se han creado tres televisoras comunitarias, sólo una con identidad cultural indígena, esta televisora tiene una sintonía del $49 \%$ de los habitantes. El bajo crecimiento de la televisión comunitaria muestra un desinterés en la creación de nuevas televisoras.

La audiencia de la televisora comunitaria indicó que el contenido refleja su identidad cultural, sin embargo, al $81 \%$ de ellos no les agradan los personajes, conductores y periodistas del canal. Para generar fidelización de los televidentes se deben considerar los siguientes aspectos: (a) en el contenido deben ver reflejada su vida diaria, (b) las dos funciones lingüísticas emotiva y apelativa que se ligan al concepto de identidad buscando representaciones e historias optimistas de la comunidad para crear valor y amor propio a su cultura (Livingston, 2003) y (c) el contenido debe estar vinculado a la audiencia y a su contexto, de esa forma genera y relaciona información eficiente (Aguaded y Cotín, 2004).

Las alianzas estratégicas con entidades que promulgan las políticas, los operadores de cable y de servicio de interface, podrían mejorar el recurso financiero de las televisoras comunitarias. Así como la ampliación de su alcance a través de la participación en medios digitales y asociaciones con canales locales para llegar a una audiencia nacional.

Las conclusiones planteadas son derivadas de resultados de estudios realizados en otros países, se sugiere realizar futuras investigaciones en Ecuador para identificar las causas del bajo crecimiento de la televisión comunitaria, las posibles alianzas estratégicas que permitan expandir su alcance y evaluar los gustos y preferencias de la audiencia de televisión comunitaria.

\section{Referencias Bibliográficas}

Agencia de Regulación y Control de las Telecomunicaciones. (2013). Ley orgánica de comunicación.
Aguaded, J., y Cotín, S. (2004). Televisión e identidad indígena una comunidad rural de La Patagonia Argentina. Comunicar, 71-79.

Aguilar, A. (2013). El diagnóstico de "laçultura organizacional o las culturas de la cultura. Global Media Journal México, 6, 67-81.

Ali, C. (2012). Media at the Margins: Policy and Practice in American, Canadian, and British Community Television. International Journal of Communication, 6 , 1119-1138.

Asamblea Nacional Repùblica del Ecuador. (2008). Constitución de la República del Ecuador.

Barrio, E. (2016, noviembre). Revista online Compromiso Empresarial.

Bañuelos, S. (2015). Análisis de programas televisivos. En B. Salvador (Ed.), Competencias comunicativas I (pp. 1-8).

Bergillos, I. (2015). Participación de la audiencia y televisión en la era digital.

Berrospi, V. (2014). El Entretenimiento Televisivo Y La Identidad Cultural, En Los Colegios Emblemáticos De La Ciudad De Huánuco.

Cabalín, C. (2010). Democracia y Comunicación. Revista F@ro-Estudios, 1-9.

Chatterjee, A. N. (2012). Inequalities in the public sphere: emergence of community television in India. Asian Ethnicity, 13, 229-250.

Contenidos sobre pueblos indígenas y afro, una deuda pendiente en medios de comunicación de Ecuador. (2014, agosto). Andes.

CORDICOM. (2016).

Fuentes-Bautista, M. (2009, enero). Beyond television: The digital transition of public access.

García, S. (2008). LA SINERGIA WEB-TELEVISIÓN: UNA NUEVA ESTRATEGIA. HOLOGRAMATICA Revista academica de la Facultad de Ciencias Sociales, 3-23.

González, M., Roncallo, S., Arango, G., y Uribe, E. (2015). Calidad en contenidos televisivos y engagement: Analisis de un canal privado en Colombia. Cuadernos Info. 
Gumucio, A. (2011). Comunicación para el cambio Social: Clave del desarrollo participativo. Signo y Pensamiento, 26-39.

Gumucio, A. (2015). Arte de equilibristas: la sostenibilidad de los medios de comunicación comunitarios. Punto Cero, 10, 6-19.

Gutiérrez, C. (2000). Televisión y calidad: Perspectivas de investigación y criterios de evaluación. Estudios de Comunicación, 5, 151-184.

Henao, H., Vergara, J., y Ortiz, E. (2014). LA TELEVISION COMUNITARIA EN EL ORIENTE ANTIOQUEÑO. UN EJERCICIO DE CONSTRUCCION DE TEJIDO SOCIAL Y PAZ. El ÁGORA USB, 14, 517-536.

Higgins, J. W. (1999). Community Television and the Vision of Media Literacy, Social Action, and Empowerment. Journal of Broadcasting \& Electronic Media, 43, 624-644.

Katsumata, H. (2012). Japanese popular culture in East Asia: a new insight into regional community building. International Relations of the Asia-Pacific, 12, 133-160.

Lee, D. H. (2004). Cultural contact with Japanese TV dramas: modes of reception and narrative transparency', in K. Iwabushi. Hong Kong: Hong Kong University Press.

Livingston, P. (2003). El totalitarismo frente a la identidad como proyecto en los medios comunitarios. Investigación \& Desarrollo, 11, 254-267.

Loor, M. (2017, septiembre). Trabajo de Graduación. Universidad de Guayaquil.

Marcús, J. (2011). Apuntes sobre el concepto de identidad. Intersticios, 5, 107-114.

Méndez, S. (2017). LA FIDELIZACIÓN EN LA TELEVISIÓN LOCAL. Estudios de Comunicación, 5, 151184.

Mora, M., y Villar, M. (2015). Los efectos de la Globalización en la identidad cultural del la etnia Mazahua. En (pp. 1-19).
Mosquera, C., Rincòn, D., y Hurtado, I. (2013). La Escuela un escenario multicultural generador de construcción dinámica de identidades.

Márquez, L. (2012). Retos de la Televisión Comunitaria en Cuba, su papel en el desarrollo global. Revista cientifca de comunicación MCHJ, 7-32.

Márquez, L. (2013). Una Tv Verdaderamente Comunitaria. Razón y Palabra, 171-180.

Ojajärvi, S., y Valtonen, S. (2012). NEWSPAPER AND TELEVISION AS LOCAL AND COMMUNITY MEDIA-ASSESSMENTS BY INDUSTRY EXPERTS. Journal of Media Business Studies, 9, 129-145.

Reyes, H. (2015). Potenciar los medios comunitarios: apuesta vital para la comunicación y la democracia. Consejo de Regulación y desarrollo de la información y comunicación.

Ruiz, C., Calderòn, I., y Torres, F. (2011). Construir la identidad en los márgenes de la globalización. Cultura y Educación, 23, 589-599.

Sandoval, L. (2009). La identidad nacional en tiempos de globalización. Revista Electrónic@Educare, XIII, 7-16.

Shahjahan, M., Chowdhury, H., Al-Hadhrami, A., y Harun, G. (2016). Antenatal and postnatal care practices among mothers in rural Bangladesh: A community based cross-sectional study. Midwifery.

SIDENPE. (2002). Sistema Integrado de Indicadores Sociales del Ecuador. Sistema de Indicadores de las Nacionalidades y Pueblos Indígenas del Ecuador.

Sánchez, A. (2012). Facultad de Educación, Artes y Humanidades. Universidad San Francisco de Paula Santander Ocaña.

Sànchez, L., y Deaza, L. (2016). La Televisión Posible: Documental Canal Comunitario TV SUESCA. Corporación Universitaria Minuto de Dios.

Sousa, A. L. N. d. (2016). Law and community television: Brazil and Argentina. Revista Eptic, 18, 127-142.

Terry, J. (2011). Cultura, Identidad Cultural, Patrimonio y Desarrollo Comunitario Rural. Contribuciones a las Ciencias Sociales, 1-28.

Recibido: 14 de febrero de 2018

Aceptado: 19 de marzo de 2018 